\title{
CORRECTIONS APPLIED TO THE CARRIER FREQUENCIES OF THE RADIO STATIONS \\ GBR AND RES ACCORDING TO THE USSR STATE STANDARD \\ OF TIME AND FREQUENCY
}

TABLE 1. Corrections Applied to the Carrier Frequencies of the Radio Stations GBR and RES According to the USSR State Standard of Time and Frequency

\begin{tabular}{|c|c|c|c|c|c|c|c|c|}
\hline \multicolumn{9}{|c|}{ October, 1970} \\
\hline Date & $\begin{array}{l}\text { GBR, } 16 \mathrm{kHz} \text { re- } \\
\text { ferred to } 03^{\mathrm{h}} \mathrm{TU}\end{array}$ & $\begin{array}{l}\text { RES, } 100 \mathrm{kHz} \text { re }- \\
\text { ferred to } 19^{\mathrm{h}} \mathrm{TU}\end{array}$ & Date & \multicolumn{2}{|c|}{$\begin{array}{l}\text { GBR, } 16 \mathrm{kHz} \text { re- } \mathrm{RES}, 100 \mathrm{kHz} \text { re } \\
\text { ferred to } 03^{\mathrm{h}} \mathrm{TU} \text { ferred to } 19^{\mathrm{h}} \mathrm{TU}\end{array}$} & Date & $\begin{array}{l}\mid \mathrm{GBR}, 16 \mathrm{kHz} \text { re - } \\
\mid \text { ferred to } 03^{\mathrm{h}} \text { TU }\end{array}$ & $\begin{array}{l}\text { RES, } 100 \mathrm{kHz} \text { re- } \\
\text { ferred to } 19^{\mathrm{h}} \mathrm{TU}\end{array}$ \\
\hline $\begin{array}{r}1 \\
2 \\
3 \\
4 \\
5 \\
5 \\
3 \\
7 \\
8 \\
9 \\
10\end{array}$ & $\begin{array}{l}-300,3.10^{-10} \\
-300,3 \\
-300,4 \\
-300,2 \\
-300,3 \\
-300,0 \\
-300,5 \\
-300,2 \\
-300,5 \\
-300,3\end{array}$ & $\begin{array}{l}-300 \cdot 3 \cdot 10^{-10} \\
-300,2 \\
-300,0 \\
-300,1 \\
-300.3 \\
-299,9 \\
-300,1 \\
-299,7 \\
-299.7 \\
-299,9\end{array}$ & $\begin{array}{l}11 \\
12 \\
13 \\
14 \\
15 \\
16 \\
17 \\
18 \\
19 \\
20\end{array}$ & $\begin{array}{l}-300,4 \cdot 10^{-10} \\
-300,1 \\
-300,4 \\
-300,3 \\
-300,3 \\
-300,4 \\
=300,4 \\
-300,5 \\
-300,4 \\
-300,5\end{array}$ & $\begin{array}{l}-299,4.10-10 \\
-300,1 \\
-300,3 \\
-300,3 \\
-300,3 \\
-300,0 \\
-300,2 \\
-300,2 \\
-300,1 \\
-299,9\end{array}$ & $\begin{array}{l}21 \\
22 \\
23 \\
24 \\
25 \\
26 \\
27 \\
28 \\
29 \\
30 \\
31\end{array}$ & $\begin{array}{l}-300,6.10^{-10} \\
-300,2 \\
-300,1 \\
-300,4 \\
-300,3 \\
-300,4 \\
-300,2 \\
-300,6 \\
-300,4 \\
-300,3 \\
-300,4\end{array}$ & $\begin{array}{l}-299,8.10^{-10} \\
-300,0 \\
-299,9 \\
-299,7 \\
-299,7 \\
-299,6 \\
-299,8 \\
-299,9 \\
-300,2 \\
-300,0\end{array}$ \\
\hline & & & & & & Mean & $-300,3$ & $-300,0$ \\
\hline
\end{tabular}

Translated from Izmeritel'naya Tekhnika, No. 1, p. 108, January, 1971.

( 1971 Consultants Bureau, a division of Plenum Publishing Corporation, 227 West 17th Street, New York, N.Y. 10011. All rights reserved. This article cannot be reproduced for any purpose whatsoever without permission of the publisher. A copy of this article is available from the publisher for $\$ 15.00$. 